\title{
Platelet to lymphocyte ratio as a prognostic factor in patients with advanced colorectal cancer undergoing palliative treatment
}

\author{
Weiwei Gui ${ }^{1}$, Xiaoyue Wang ${ }^{1}$, Yizhou Luo ${ }^{1}$, Jian Wang ${ }^{2}$ \\ ${ }^{1}$ Department of Oncology, The Air Force Hospital from Eastern Theater of PLA, Nanjing, China; ${ }^{2}$ Department of Oncology, The Fifth People's \\ Hospital of Changshu, Changshu, China \\ Contributions: (I) Conception and design: J Wang; (II) Administrative support: Y Luo; (III) Provision of study materials or patients: W Gui; (IV) \\ Collection and assembly of data: W Gui, X Wang; (V) Data analysis and interpretation: X Wang, Y Luo; (VI) Manuscript writing: All authors; (VII) \\ Final approval of manuscript: All authors. \\ Correspondence to: Jian Wang, P.D. Department of Oncology, The Fifth People's Hospital of Changshu, Changshu 215500, China. \\ Email: wangj020@hotmail.com.
}

Background: Our study aims to investigate the level of platelet to lymphocyte ratio (PLR) and determine its prognostic value in patients with advanced colorectal cancer undergoing palliative treatment.

Methods: One hundred and fifty-two patients with advanced colorectal cancer confirmed in our hospital from January 2013 to January 2018 were selected as study participants. The boundary-value of PLR was determined by receiver operating characteristics (ROC) curves. Furthermore, the relationship between PLR and clinical characteristics of patients with advanced colorectal cancer was analyzed. Next, the prognostic factors affecting the survival time were analyzed by Kaplan-Meier single factor survival analysis and Cox multivariate regression model.

Results: According to the ROC curve, the optimal critical value of PLR was 207.29. Patients were divided into high PLR ( $\mathrm{n}=73$ ) and low PLR (n=79) groups. The median survival time was $68.0(24.5,296.5)$ days for the high PLR group, and 124 (34, 438) days for the low PLR group and differences between the groups were statistically significant $(\mathrm{P}<0.05)$. Both groups had similar demographic features, namely gender, age, Eastern Cooperative Oncology Group (ECOG) score, and several metastasis sites ( $\mathrm{P}>0.05)$. Albumin and hemoglobin levels were found to be negatively correlated to PLR $(\mathrm{P}<0.05)$. Cox multivariate regression model results showed that PLR, albumin, and ECOG score were independent prognostic factors $(\mathrm{P}<0.05)$.

Conclusions: This study demonstrated that PLR is an independent prognostic factor of survival time, with good predictive value, in patients with advanced colorectal cancer undergoing palliative treatment. High PLR was significantly correlated to reduced survival rates, while low PLR was associated with better longterm survival rates.

Keywords: Colorectal cancer; platelet to lymphocyte ratio (PLR); prognostic

Submitted Jun 16, 2020. Accepted for publication Aug 25, 2020.

doi: 10.21037/apm-20-1389

View this article at: http://dx.doi.org/10.21037/apm-20-1389

\section{Introduction}

The incidence rate of colorectal cancer is third among all cancers worldwide, while the rate of mortality is second only to lung cancer (1). In 2018, there were approximately 1.8 million new cases and 880,000 deaths due to colorectal cancer globally. Although many treatments have improved the prognosis of colorectal cancer, the situation remains unfavorable. In particular, patients with advanced colorectal cancer who have previously undergone surgery or chemotherapy still have a limited total survival time. As a result of previous treatment experiences, some patients prefer palliative care in terminal stages as their demand for an improved quality of life increases. In recent years, hospice care has been paid more and more attention. 
Palliative treatment as a part of hospice care is mainly to relieve symptoms, relieve pain and improve the quality of life. The treatment methods mainly include the treatment of symptoms affecting the quality of life such as pain, anorexia and fatigue, as well as mental and psychological intervention. The object of our study is colorectal patients received palliative treatment, as clinical attention and research to these patients were less. But the number of these patients is not small. Thus, accurate biomarkers that evaluate the prognosis of these patients are necessary to support patients and their families, and provide information so that they may make timely, informed plans and health decisions.

Previous studies have identified tumor size, clinical stage, and tumor indexes as the critical prognostic factors for colorectal cancer (2-5). Lately, KPS, number of brain metastases, the albumin-to-globulin ratio, fibrinogen-toalbumin ratio and other hematological indicators are proved to related to overall survival in patients with colorectal cancer. In recent years, many studies have confirmed that the occurrence and metastasis of malignant tumors are related to inflammation and immune response (6-9). It has been shown that platelets, lymphocytes, neutrophils, C-reactive protein, and Glasgow prognostic score-as indicators of inflammation and immune response-have prognostic value in a variety of cancers. Furthermore, the platelet to lymphocyte ratio (PLR) - as one of the indexes to evaluate the balance of inflammatory and immune response-has been demonstrated to be useful for the prognosis of the lung, gastric, colorectal, and other cancers (10-13). However, the prognostic value of PLR in the palliative treatment of advanced colorectal cancer is rarely reported.

Therefore, the purpose of this study is to investigate the relationship between PLR level and survival in patients with advanced colorectal cancer receiving palliative treatment and exploring the clinical value of PLR in predicting the prognosis of advanced colorectal cancer.

We present the following article in accordance with the MDAR reporting checklist (available at http://dx.doi. org/10.21037/apm-20-1389).

\section{Methods}

\section{Patients}

One hundred fifty-two patients with advanced colorectal cancer who received palliative treatment from January 2013 to January 2018 in The Air Force Hospital from Eastern Theater of PLA were selected as the study participants. Patients were eligible for inclusion in the study based on the following criteria: (I) age between 18 and 90 years old; (II) colorectal cancer confirmed by histopathology; (III) determined as stage IV according to the $7^{\text {th }}$ edition of the American Joint Committee on Cancer (AJCC) staging system for colorectal cancer; (IV) progress following previous surgery or chemotherapy; and (V) no radiotherapy, chemotherapy, or targeted treatment in the three months before the study. Patients were excluded from the study based on the following: (I) patients previously diagnosed with chronic diseases (such as coronary heart disease, hypertension, and chronic obstructive pulmonary disease); (II) those with acute and chronic infectious diseases; (III) those with autoimmune diseases; (IV) those with other malignant cancers and blood system diseases; (V) patients who had used antibiotics, hormones, or anticoagulants within the three months before the study; and (VI) patients with incomplete clinical data. All patients received palliative treatment. Palliative treatment includes analgesia, antiemetic, nutritional support, psychotherapy and so on. All patients participating in the study signed the informed consent. The study was approved by the hospital ethics committee, and was in line with medical ethics standards according to the Helsinki Declaration of the World Medical Association.

\section{Study methods}

Clinical characteristics from medical records were collected and analyzed and included sex, age, number of tumor metastasis sites, and ECOG score. After admission, platelet count, Lymphocyte count, hemoglobin, and albumin from routine blood and biochemical examination were collected. The ratio of platelet to lymphocyte was represented as PLR. The total survival time was defined as the time from diagnosis of stage IV to death due to colorectal cancer. All cases were followed for up to two years from the joining date. The final follow-up date was January 1, 2020, and the follow-up rate was $100 \%$.

\section{Statistical analysis}

Spss 22.0 software was used for data processing and analysis. Receiver operating characteristic (ROC) curve analysis was performed to confirm the Youden Index and to subsequently identify the optimal cutoff value for PLR according to the 
Table 1 Baseline patient characteristics

\begin{tabular}{lc}
\hline Baseline patient characteristics & Value \\
\hline Sex & \\
Male & $81(53.3 \%)$ \\
Female & $71(46.7 \%)$ \\
Age, years & $60.1( \pm 13.2)$ \\
$<60$ & $77(50.7 \%)$ \\
$\geq 60$ & $75(49.3 \%)$ \\
Number of metastases & \\
$>1$ & $80(52.6 \%)$ \\
1 & $72(47.4 \%)$ \\
ECOG score & \\
$>2$ & $68(44.7 \%)$ \\
$\leq 2$ & $84(52.6 \%)$ \\
Albumin, g/L & $33.1( \pm 6.7)$ \\
$<35$ & $80(52.6 \%)$ \\
$\geq 35$ & $72(47.4 \%)$ \\
Hemoglobin, g/L & $107.91( \pm 23.37)$ \\
$<115$ & $93(61.2 \%)$ \\
PLR & $59(38.8 \%)$ \\
\hline PLR & \\
\hline 115 & $04.72(140.38,329.32)$ \\
\hline
\end{tabular}

PLR, platelet to lymphocyte ratio.

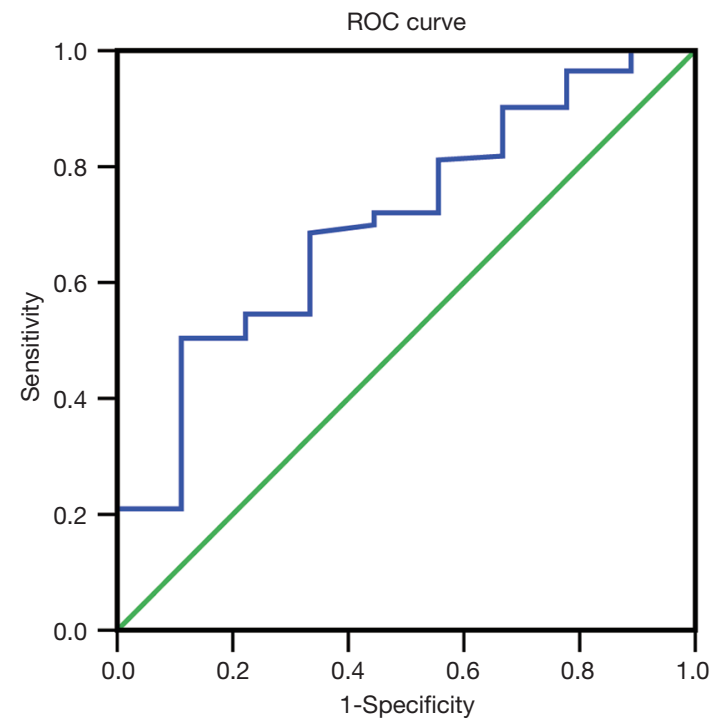

Figure 1 ROC curves for PLR. AUC, 0.706; 95\% CI: 0.542-0.869, $\mathrm{P}=0.039$. ROC, receiver operating characteristic; PLR, platelet to lymphocyte ratio. area under the ROC curve (AUC). The mean \pm standard deviation was used for normally distributed measurement data, and the median (interquartile range) [M (P25, P75)] was used when the measurement data were non-normally distributed. Counting data was expressed by a numerical value, and the chi-square $\left(\chi^{2}\right)$ test was used for comparison between different groups (which were from categorical data). The Kaplan-Meier method and the log-rank test were used to analyze survival factors and compare the survival differences between high and low PLR groups. Univariate and multivariate Cox regressions analysis was used to determine the independent prognostic factor affecting long-term survival. The hazard ratio (HR) and 95\% CI was reported from the multivariate Cox analysis. A two-sided $\mathrm{P}$ value less than 0.05 was considered statistically significant.

\section{Results}

\section{Baseline patient characteristics}

A total of 152 patients were enrolled in this study. Table 1 shows the baseline characteristics of all patients. There were 81 males $(53.3 \%)$ and 71 females (46.7\%). The mean age was $60.1( \pm 13.2)$ years. The mean value of PLR was 204.72 (140.38, 329.32). The mean survival time was 101 (31.25, 325.75 ) days. The 30,60 , and 180 -day rate of 152 patients were $51.97 \%, 36.18 \%$, and $19.74 \%$, respectively.

\section{Patient characteristics and survival in High and low PLR groups}

ROC curve analysis of PLR in these patients showed that the AUC of PLR was 0.706 (Figure 1). The sensitivity was 0.505 ; the specificity was 0.889 ; the best cutoff value was 207.29. Patients were divided into a high PLR group (PLR 2207.29 ) and a low PLR group (<207.29), as shown in Table 2. In all patients, the rate of the high PLR group was $48.7 \%$ (73/152), while the low PLR group was $52.3 \%$ (79/152). There was no statistically significant difference between the two groups in age, gender, ECOG score, and the number of metastasis sites $(\mathrm{P}>0.05)$; however, there were statistically significant differences in hemoglobin and albumin levels $(\mathrm{P}<0.05)$. The median survival time of the high PLR group was 68 and 126 days for the low PLR group. Statistical differences between these groups are shown in Figure 2. Ninety-, 180-, and 360-day survival rates for the low PLR group were: $58.2 \%, 44.3 \%$, and $29.1 \%$, and were $46.6 \%$, $31.5 \%$ and $13.7 \%$ for the high PLR group (data not shown). 
Table 2 Distribution of variables in high and low PLR groups

\begin{tabular}{|c|c|c|c|}
\hline Variables & $\begin{array}{l}\text { High PLR } \\
(\geq 207.29)\end{array}$ & $\begin{array}{l}\text { Low PLR } \\
(<207.29)\end{array}$ & $P$ value \\
\hline \multicolumn{4}{|l|}{ Sex } \\
\hline Male & 38 & 43 & 0.769 \\
\hline Female & 35 & 36 & \\
\hline \multicolumn{4}{|c|}{ Age, years } \\
\hline$<60$ & 38 & 39 & 0.741 \\
\hline$\geq 60$ & 35 & 40 & \\
\hline \multicolumn{4}{|c|}{ Number of metastases } \\
\hline 1 & 36 & 36 & 0.644 \\
\hline$>1$ & 37 & 43 & \\
\hline \multicolumn{4}{|c|}{ ECOG score } \\
\hline$<3$ & 38 & 46 & 0.444 \\
\hline$\geq 3$ & 35 & 33 & \\
\hline \multicolumn{4}{|c|}{ Albumin, g/L } \\
\hline$<35$ & 45 & 35 & 0.032 \\
\hline$\geq 35$ & 28 & 44 & \\
\hline \multicolumn{4}{|c|}{ Hemoglobin, g/L } \\
\hline$<115$ & 52 & 40 & 0.009 \\
\hline$\geq 115$ & 21 & 39 & \\
\hline
\end{tabular}

PLR, platelet to lymphocyte ratio.

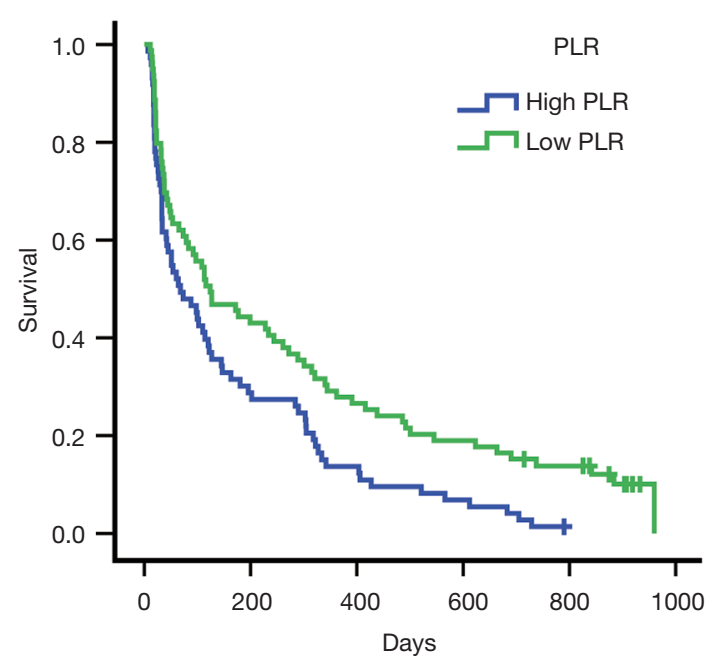

Figure 2 Kaplan-Meier curves of PLR according to survival time, $\mathrm{P}=0.006$ (log-rank test). PLR, platelet to lymphocyte ratio.

\section{Univariate and multivariate analysis of prognostic survival in patients with colorectal cancer}

Kaplan-Meier single factor survival analysis and the logrank test results showed that ECOG score, hemoglobin, albumin, and PLR were prognostic factors of survival time in patients with advanced colorectal cancer $(\mathrm{P}<0.05)$. Cox multivariate regression model analysis showed that ECOG score, albumin, and PLR were independent prognostic factors of survival time in patients with advanced colorectal cancer $(\mathrm{P}<0.05)$ (see Table 3).

\section{Discussion}

Previous studies have demonstrated that inflammation is related to tumor progression. The physiopathological mechanism between inflammation and tumor progression is still unknown; however, several theories may explain it. The local environment of tumor growth (i.e., the tumor microenvironment) could lead to tumor proliferation, invasion, and metastasis $(14,15)$. The primary cell components of a tumor microenvironment are neutrophils, lymphocytes, platelets, macrophages, and natural killer cells, among others. In recent years, studies have shown that platelets from peripheral blood were collected into a tumor microenvironment through blood circulation, and participated in a chronic inflammatory response. Meanwhile, tumor cells can stimulate platelet activation by directly contacting or releasing platelet-inducing factors, such as adenosine diphosphate (ADP). ADP activates tumor cell-surface receptors and induces secretion of a variety of bioactive substances to affect cell and vascular growth. They also promote swelling, tumor cell growth, invasion, migration, exudation, and distant metastasis (16-18). Lymphocytes were the principal cells of characteristic immunity. In particular, CD4+ and CD8+T lymphocytes act in unison to form the immune barrier and kill tumor cells $(19,20)$. Adenosine (produced by tumor cells) and lactic acid (produced by glycolysis) can inhibit the proliferation and function of effector $\mathrm{T}$ lymphocytes (21). Thus, the PLR can reflect the balance of inflammation and host immune reaction and provide insight regarding tumor diagnosis and prognosis evaluation

Numerous studies have established the correlation between higher PLR and poor long-term outcomes for many cancers, including lung (10), gastric (11), and breast (13), among others. Moreover, the results obtained in some studies of colorectal cancer concurred with our results $(22,23)$, 
Table 3 Univariate and multivariate analysis of prognostic survival

\begin{tabular}{lccc}
\hline Variables & $\begin{array}{c}\text { Univariate analysis } \\
P \text { value }\end{array}$ & Multivariate analysis \\
\hline Sex & 0.093 & & H value $(95 \% \mathrm{Cl})$ \\
Age & 0.175 & & \\
Number of metastases & 0.428 & & 0.000 \\
EOCG score & 0.000 & $2.183(1.528-3.120)$ & 0.001 \\
Albumin & 0.000 & $2.055(1.360-3.106)$ & 0.580 \\
Hemoglobin & 0.000 & $1.124(0.742-1.703)$ & 0.024 \\
PLR & 0.006 & $0.676(0.480-0.950)$ & \\
\hline
\end{tabular}

PLR, platelet to lymphocyte ratio.

and some meta-analyses came to similar conclusions (24). This study employed a ROC analysis to define the optimal cutoff PLR value. Previous studies reported that the cutoff value in colorectal patients was $153-216(25,26)$, which was similar to the values observed in our study (PLR $=207.29$ ). Low PLR was associated with long-term survival. Univariate and multivariate Cox proportional-hazard analysis revealed that PLR was an independent prognostic factor for survival time in patients with colorectal cancer. In our study, PLR showed no correlation with sex, age, and ECOG score, though it was strictly correlated to albumin and hemoglobin levels. Similar observations have been reported by other studies, indicating that both albumin and hemoglobin may influence immunological function, though the mechanism underlying their interaction is unknown at this stage. Despite the complex relationship between these factors, it is reasonable to assume that they are likely related to cancer progression.

Albumin and hemoglobin were indexes of nutritional status. There are some reports about the relationship between albumin and hemoglobin and the survival rate of certain cancers, though their conclusions differ. Man et al. showed that pre-treatment albumin level was an independent indicator for overall survival (27), while Li et al. investigated 312 patients with non-metastatic colon cancer, and reported that albumin and hemoglobin were not independent prognostic indicators for overall survival (28). Our study assessed the value of albumin and hemoglobin in advanced colorectal cancer. Univariate analysis showed that albumin and hemoglobin were associated with survival time, with high albumin and hemoglobin levels being positively correlated with long-term survival. However, multivariate Cox proportional-hazard analysis demonstrated that although albumin was an independent prognostic factor, hemoglobin was not, which is congruent with the results observed by Man et al. but differs from that observed by Li et al. Also, the ECOG score was found to be an independent prognostic indicator for survival time. A possible explanation for this could be that increased activity leads to higher food intake, which is related to nutritional status.

There are several limitations to this study that should be acknowledged. Firstly, the sample size was limited. Secondly, this study is based on published reports. Finally, this is a one-center study and lacks a multi-center comparison. Hence, in order to reliably confirm the results obtained in this study, prospective research on much larger sample size is required.

In conclusion, this study demonstrated that PLR is an independent prognostic factor of survival time in patients with advanced colorectal cancer undergoing palliative treatment. High PLR was significantly correlated to poor survival rates, while low PLR was associated with a better long-term survival rate. PLR exhibits strong predictive power in patients with advanced colorectal cancer undergoing palliative treatment, and can be useful in supporting patients and their families to make timely, informed plans and health decisions.

\section{Acknowledgments}

We would like to thank Xian Xu for her useful suggestions. Funding: None.

\section{Footnote}

Reporting Checklist: The authors have completed the MDAR 
reporting checklist. Available at http://dx.doi.org/10.21037/ apm-20-1389

Data Sharing Statement: Available at http://dx.doi. org/10.21037/apm-20-1389

Conflicts of Interest: All authors have completed the ICMJE uniform disclosure form (available at http://dx.doi. org/10.21037/apm-20-1389). The authors have no conflicts of interest to declare.

Ethical Statement: The authors are accountable for all aspects of the work in ensuring that questions related to the accuracy or integrity of any part of the work are appropriately investigated and resolved. All patients participating in the study signed the informed consent. The study was approved by the hospital ethics committee, and was in line with medical ethics standards according to the Helsinki Declaration of the World Medical Association (20200701001).

Open Access Statement: This is an Open Access article distributed in accordance with the Creative Commons Attribution-NonCommercial-NoDerivs 4.0 International License (CC BY-NC-ND 4.0), which permits the noncommercial replication and distribution of the article with the strict proviso that no changes or edits are made and the original work is properly cited (including links to both the formal publication through the relevant DOI and the license). See: https://creativecommons.org/licenses/by-nc-nd/4.0/.

\section{References}

1. Bray F, Ferlay J, Soerjomataram I, et al. Global cancer statistics 2018: GLOBOCAN estimates of incidence and mortality worldwide for 36 cancers in 185 countries. CA Cancer J Clin 2018;68:394-424.

2. Liu F, Zhao J, Li C, et al. The unique prognostic characteristics of tumor deposits in colorectal cancer patients. Ann Transl Med 2019;7:769.

3. Wu G, Li J, Qin C. Reduced RANBP9 expression is associated with poor prognosis in colorectal cancer patients. Transl Cancer Res 2019;8:2704-12.

4. Huang B, Chen C, Ni M, et al. The association between small tumor size and poor survival in $\mathrm{T} 4$ mucinous adenocarcinoma of colon without distant metastasis. J BUON 2017;22:170-7.

5. Abdel-Rahman O, Cheung WY. Revisiting the prognostic relevance of muscle mass among non-metastatic colorectal cancer. Transl Gastroenterol Hepatol 2018;3:55.

6. Murata M. Inflammation and cancer. Environ Health Prev Med 2018;23:50.

7. Singh N, Baby D, Rajguru JP, et al. Inflammation and cancer. Ann Afr Med 2019;18:121-6.

8. Singh R, Mishra MK, Aggarwal H. Inflammation, Immunity, and Cancer. Mediators Inflamm 2017;2017:6027305.

9. Todoric J, Antonucci L, Karin M. Targeting Inflammation in Cancer Prevention and Therapy. Cancer Prev Res (Phila) 2016;9:895-905.

10. Amaral SR, Casal Moura M, Carvalho J, et al. Prognostic significance of neutrophil-to-lymphocyte ratio (NLR) and platelet-to-lymphocyte ratio (PLR) in non-small cell lung cancer (NSCLC) treated with immune checkpoint inhibitors. Ann Oncol 2019. doi:10.1093/annonc/mdz063.

11. Chen L, Hao Y, Cong X, et al. Peripheral Venous Blood Platelet-to-Lymphocyte Ratio (PLR) for Predicting the Survival of Patients With Gastric Cancer Treated With SOX or XELOX Regimen Neoadjuvant Chemotherapy. Technol Cancer Res Treat 2019;18:1533033819829485.

12. Cruz-Ramos M, Del Puerto-Nevado L, Zheng B, et al. Prognostic significance of neutrophil-to lymphocyte ratio and platelet-to lymphocyte ratio in older patients with metastatic colorectal cancer. J Geriatr Oncol 2019;10:742-8.

13. Cuello-López J, Fidalgo-Zapata A, LópezAgudelo L, et al. Platelet-to-lymphocyte ratio as a predictive factor of complete pathologic response to neoadjuvant chemotherapy in breast cancer. PLoS One 2018;13:e207224.

14. Denton AE, Roberts EW, Fearon DT. Stromal Cells in the Tumor Microenvironment. Adv Exp Med Biol 2018;1060:99-114.

15. Yang L, Lin PC. Mechanisms that drive inflammatory tumor microenvironment, tumor heterogeneity, and metastatic progression. Semin Cancer Biol 2017;47:185-95.

16. Ma X, Wang Y, Sheng H, et al. Prognostic significance of thrombocytosis, platelet parameters and aggregation rates in epithelial ovarian cancer. J Obstet Gynaecol Res 2014;40:178-83.

17. Camerer E, Qazi AA, Duong DN, et al. Platelets, proteaseactivated receptors, and fibrinogen in hematogenous metastasis. Blood 2004;104:397-401.

18. Michael JV, Wurtzel JGT, Mao GF, et al. Platelet microparticles infiltrating solid tumors transfer miRNAs that suppress tumor growth. Blood 2017;130:567-80. 
19. Farhood B, Najafi M, Mortezaee K. CD8+cytotoxic T lymphocytes in cancer immunotherapy: A review. J Cell Physiol 2019;234:8509-21.

20. Ostroumov D, Fekete-Drimusz N, Saborowski M, et al. CD4 and CD8 T lymphocyte interplay in controlling tumor growth. Cell Mol Life Sci 2018;75:689-713.

21. Sakai C, Nishikawa H. Immunosuppressive Environment in Tumors. Gan To Kagaku Ryoho 2018;45:222-6.

22. Krakowska M, Dębska-Szmich S, Czyżykowski R, et al. The prognostic impact of neutrophil-to-lymphocyte ratio, lymphocyte-to-monocyte ratio, and platelet-to-lymphocyte ratio in patients with advanced colorectal cancer treated with first-line chemotherapy. Prz Gastroenterol 2018;13:218-22.

23. Hu Z, Tan S, Chen S, et al. Diagnostic value of hematological parameters platelet to lymphocyte ratio and hemoglobin to platelet ratio in patients with colon cancer. Clin Chim Acta 2020;501:48-52.

24. Min GT, Wang YH, Yao N, et al. The prognostic role of pretreatment platelet-to-lymphocyte ratio as predictors in patients with colorectal cancer: a meta-analysis. Biomark Med 2017;11:87-97.

25. Zhang J, Zhang HY, Li J, et al. The elevated NLR, PLR and PLT may predict the prognosis of patients with colorectal cancer: a systematic review and meta-analysis. Oncotarget 2017;8:68837-46.

26. Bailon-Cuadrado M, Choolani-Bhojwani E, Tejero-Pintor FJ, et al. Preoperative platelet-lymphocyte ratio is an independent factor of poor prognosis after curative surgery for colon cancer. Updates Surg 2018;70:33-9.

27. Man YN, Chen YF. Systemic immune-inflammation index, serum albumin, and fibrinogen impact prognosis in castration-resistant prostate cancer patients treated with first-line docetaxel. Int Urol Nephrol 2019;51:2189-99.

28. Li Z, Xu Z, Huang Y, et al. Prognostic values of preoperative platelet-to-lymphocyte ratio, albumin and hemoglobin in patients with non-metastatic colon cancer. Cancer Manag Res 2019;11:3265-74.

(English Language Editor: A. Kassem and J. Chapnick)
Cite this article as: Gui W, Wang X, Luo Y, Wang J. Platelet to lymphocyte ratio as a prognostic factor in patients with advanced colorectal cancer undergoing palliative treatment. Ann Transl Med 2020;9(5):3271-3277. doi: 10.21037/apm-20-1389 